\title{
Impact of Family YMCA Membership on Childhood Obesity: A Randomized Controlled Effectiveness Trial
}

\author{
Maurice Duggins, MD, Phillip Cherven, MD, Joe Carrithers, PhD, \\ Janet Messamore, BSN, MN, ARNP, and Annie Harvey, PhD
}

Background: Treatment studies about childhood obesity in primary care are lacking. We hypothesized that providing a paid family membership to the YMCA would be effective in reducing weight.

Methods: Patients 5 to17 years old in at least the 85th body mass index (BMI) percentile were eligible. All participants were scheduled to attend 4 nutrition classes and to return for evaluation at $2,4,6$, 9 , and 12 months. Participants were randomized to nutrition classes only $(n=39)$ or nutrition classes and family YMCA membership $(n=44)$. The primary outcome measure was year change in BMI-for-age percentile.

Results: Median BMI percentile at baseline was 99. Only 27 of 36 evaluable participants in the treatment group visited the YMCA. Four participants in the control group and one in the treatment group achieved the target reduction of $2 \mathrm{BMI}$ percentile points (Fisher's exact, $P=.17$ ). Within the treatment group, YMCA attendees had a mean increase of $0.30 \mathrm{BMI}$ points compared with an increase of $0.60 \mathrm{BMI}$ points in nonattendees $(P=.28)$.

Conclusion: In very obese children, eliminating financial barriers to YMCA membership is insufficient to induce more weight loss during 1 year compared with nutrition classes alone. Improvements in nutrition intake were reported by both groups. (J Am Board Fam Med 2010;23:323-333.)

Keywords: Obesity, Child, Pediatrics, Exercise, Body Mass Index

Childhood obesity is a major public health problem in the United States. Data from National Health And Nutrition Examination Surveys (NHANES 1976 to 1980 and 2003 to 2006) show that the prevalence of obese children (ie, those at or above the 95th percentile of the Centers for Disease Control and Prevention growth charts) has increased: for those aged 6 to 11 years, prevalence increased from $6.5 \%$ to $17.0 \%$; for those aged 12 to 19 years,

This article was externally peer reviewed.

Submitted 31 December 2008; revised 8 December 2009; accepted 14 December 2009.

From the Department of Family and Community Medicine (MD) and the Department of Pediatrics (PC), University of Kansas School of Medicine-Wichita; the Via Christi Family Medicine Residency (MD), the Via Christi Health Research Department (JC, AH), and the Via Christi Health Family Medicine Clinics (PC, JM), Wichita, KS.

Funding: Funding was provided by K. T. Wiedemann Foundation, Children's Miracle Network, Medical Society of Sedgwick County, and the Greater Wichita YMCA.

Conflict of interest: none declared.

Corresponding author: Maurice Duggins, MD, Family Medicine Residency Program, University of KansasWichita, 1121 S. Clifton, Wichita, KS 67218 (E-mail: maurice_duggins@via-christi.org). prevalence increased from $5.0 \%$ to $17.6 \%{ }^{1}$ Obesity and overweight (body mass index [BMI] percentile 85 to 94) among children and adolescents can result in a variety of adverse health outcomes, including type 2 diabetes, obstructive sleep apnea, hypertension, dyslipidemia, and the metabolic syndrome. ${ }^{2} \mathrm{Up}$ to $80 \%$ of overweight adolescents may become obese adults, ${ }^{2}$ and successful efforts to reduce the incidence of childhood obesity may reduce adult obesity and its associated diseases. ${ }^{3}$

Evidence from the most recent Cochrane review $^{4}$ shows that family-based lifestyle interventions, with a behavioral program aimed at changing thinking patterns about diet and physical activity, provide a significant and clinically meaningful decrease in overweight among both children and adolescents compared with standard care or self-help in the short and the long terms. Dietary patterns are typically addressed through education; the American Heart Association recommends the use of a registered dietitian to provide nutritional education. ${ }^{5}$ In addition to addressing diet, including exercise in treatment improves outcomes over nu- 
tritional education alone. ${ }^{6,7}$ Family-based behavioral interventions are most effective in treatment of overweight children; Epstein et $\mathrm{al}^{6}$ demonstrated maintenance of treatment effects over 10 years when parents were included as active participants in treatment.

Unfortunately, treatment studies have largely been conducted in academic centers and have yet to be translated to primary care. Primary care physicians are expected to screen for and address this health problem, ${ }^{8}$ and the US Preventive Services Task Force has called for effective clinical approaches for the treatment of overweight among children that can be implemented by primary care clinicians. ${ }^{9}$ An expert committee convened by the American Medical Association (AMA) ${ }^{8}$ recommends that treatment of obese children use a staged approach, beginning with brief counseling that can be delivered in a health care office, with the goal of instilling permanent healthy lifestyle habits.

We sought to test the effectiveness of an evidence-based intervention that feasibly could be incorporated into the routine primary care of a diverse population. A randomized, controlled trial was designed to examine weight change among children identified as obese or overweight after receiving a physician-prescribed, free 1-year family membership to a local YMCA. Because expense has been identified as the primary barrier to participation in physical activities, ${ }^{10}$ we hypothesized that eliminating financial barriers by this simple, physician-recommended approach would be effective in encouraging increased physical activity in the environment of a supportive family.

\section{Methods}

This study was designed to address the pragmatic question of whether eliminating financial barriers to YMCA membership leads to weight loss in the context of routine clinical care. As such, inclusion criteria were broad, standard clinical measures were used, and the interventions were structured as they might be in usual clinical care.

\section{Study Participants}

The study was conducted in 2 Family Medicine Clinics and a specialty Pediatrics Clinic of Via Christi Regional Medical Center. Patients in these Midwestern, urban, residency program clinics represented a wide variety of socioeconomic back- grounds. After review and approval by the institutional review board, children and adolescents 5 to 17 years old with a BMI at or above the 85th percentile for age and sex were identified during regular office visits and were referred to the study coordinator at the discretion of clinic physicians. There were no criteria for exclusion. Materials (informed consent/assent, handouts, nutrition classes) were available in both English and Spanish to accommodate families speaking Spanish only. A parent or guardian of each participant provided voluntary informed consent, and each participant read (or had read to them) and signed an assent form before being randomized into this study.

\section{Randomization}

The study physician enrolled participants using a computer-generated randomization list. The allocation sequence was concealed before randomization by using sequentially numbered envelopes containing the group-appropriate materials. Given the nature of the intervention neither clinicians nor participants were blind to the treatment allocation once randomization occurred.

\section{Procedures}

At enrollment every family received a handbook from the Center for Disease Control and Prevention's program We Can!, or Ways to Enhance Children's Activity and Nutrition. This program was designed to help children 8 to 13 years old stay at a healthy weight through improving food choices, increasing physical activity, and reducing time spent on a computer or watching TV. Studyrelated visits were scheduled for all participants at 2 months, 4 months, 6 months, 9 months, and 12 months after enrollment to evaluate physical condition since the last appointment, provide emotional support and reassurance, and answer any questions.

Every participant and their parents or guardians were scheduled to attend 4 nutrition classes, held after school and work hours, irrespective of their treatment group. These were project-exclusive, dietitian-led classes during which proper diet, nutrition, eating habits, and meal planning were discussed. Classes did not differentiate between those in the control and treatment groups, and the dietitian was given no indication of group assignment. The first class was scheduled within the 6 weeks after enrollment; the second class 1 week after the 
first class. A third nutrition class was scheduled during the 6-month visit and included preparation of healthy snacks for consumption. A fourth class was scheduled during the 9-month visit. Eating habits were surveyed at the first and the fourth class; perceptions of the project were elicited during the fourth class. Study-related physician visits and nutrition classes were provided at no cost to participants.

Families were randomized to one of 2 groups: nutrition classes only (control group) or nutrition classes and YMCA membership (treatment group). The treatment group was provided a 1 -year, nocost family membership to any of 6 area YMCAs. The YMCAs were located in different quadrants of the city as well as a centralized location. Activities offered at all the YMCAs included in the study were aquatics such as swimming and water aerobics, a track for walking or jogging, and weights (for adolescents) in a variety of sizes. At the facility the entire family could participate in the same activities or different activities during the same family visit. The first visit to the YMCA was prescribed by the physician and scheduled with telephone reinforcement by study personnel within 2 weeks of study enrollment. At this initial visit the participant, family members, and a study co-coordinator met with YMCA staff, who provided an orientation and answered any questions or concerns. YMCA diaries were completed by the participant during each visit to the YMCA throughout the 12-month study duration. YMCA staff was alerted by their computer system when a participant visited, and they reminded the participant to fill out their diary before leaving.

\section{Outcome Measurements}

Participants' height and weight were collected and entered into the medical record at baseline and at 2 months, 4 months, 6 months, 9 months, and 12 months after enrollment by the nonblinded nursing staff. Staff were instructed to obtain weights on a digital scale with participants wearing patient gowns and no footwear. Heights were taken in triplicate with a stadiometer and no footwear. BMIfor-age percentile was determined using measured height and weight and reference to age- and sexnormative data from the Centers for Disease Control and Prevention. ${ }^{11}$

Between the ages of 5 and 17, maintenance of BMI during a 1-year interval leads to a decline of roughly 2 BMI percentile points. This led us to choose as our goal a decrease of 2 points in the primary outcome measure-BMI percentilewhich was readily explained visually to parents. A secondary outcome measure was meeting the AMA Expert Committee ${ }^{8}$ weight loss targets, which differ by age and obesity status. AMA targets for the participants we enrolled call for weight maintenance (which we further defined as no more than a 5-lb gain during the 1-year study period) or weight loss.

Attendance at nutrition classes was tracked by study personnel, who signed participants into each session. Eating habits were assessed by questionnaire during the first and fourth nutrition classes. Participants reported their usual type (scored 1 for nutrient-dense to 3 for caloriedense) and number of servings (eg, 0, 1 to 2, 3 to $4,5+)$ of each of the 8 "Go, Slow, Whoa" food groups. ${ }^{12}$ For example, in the food group "beverages," the Go food types ("water, fat-free milk, or $1 \%$ low-fat milk; diet soda; unsweetened ice tea or diet iced tea, and lemonade") were scored as a 1 ; the Slow food types (" $2 \%$ low-fat milk; $100 \%$ fruit juice; sports drinks") were scored as a 2; and the Whoa food types ("whole milk; regular soda; calorically sweetened iced teas and lemonade; fruit drinks with less than 100\% fruit juice") were scored as a 3. Average baseline and exit scores for food type and number of servings were determined.

YMCA attendance was tracked electronically by each YMCA. The activities and time spent on them were recorded on a paper diary by participants.

\section{Data Analysis}

Overall attendance at scheduled study-related visits was poor: only 2 participants in each group attended all 6 scheduled visits. Consequently, so long as height and weight were recorded, other visits to the clinic within the respective visit interval were also used to follow changes in BMI.

Participants without at least baseline and one record of BMI data after baseline were excluded from analysis. For the remaining participants, values after intervention were determined on a lastobservation-carried-forward basis. $\chi^{2}$ tests were used to test differences in proportions and nonparametric Mann-Whitney tests were used to assess between-group differences. 
Figure 1. Participant flow diagram comparing nutrition class only to nutrition class plus family membership to the YMCA. BMI, body mass index.

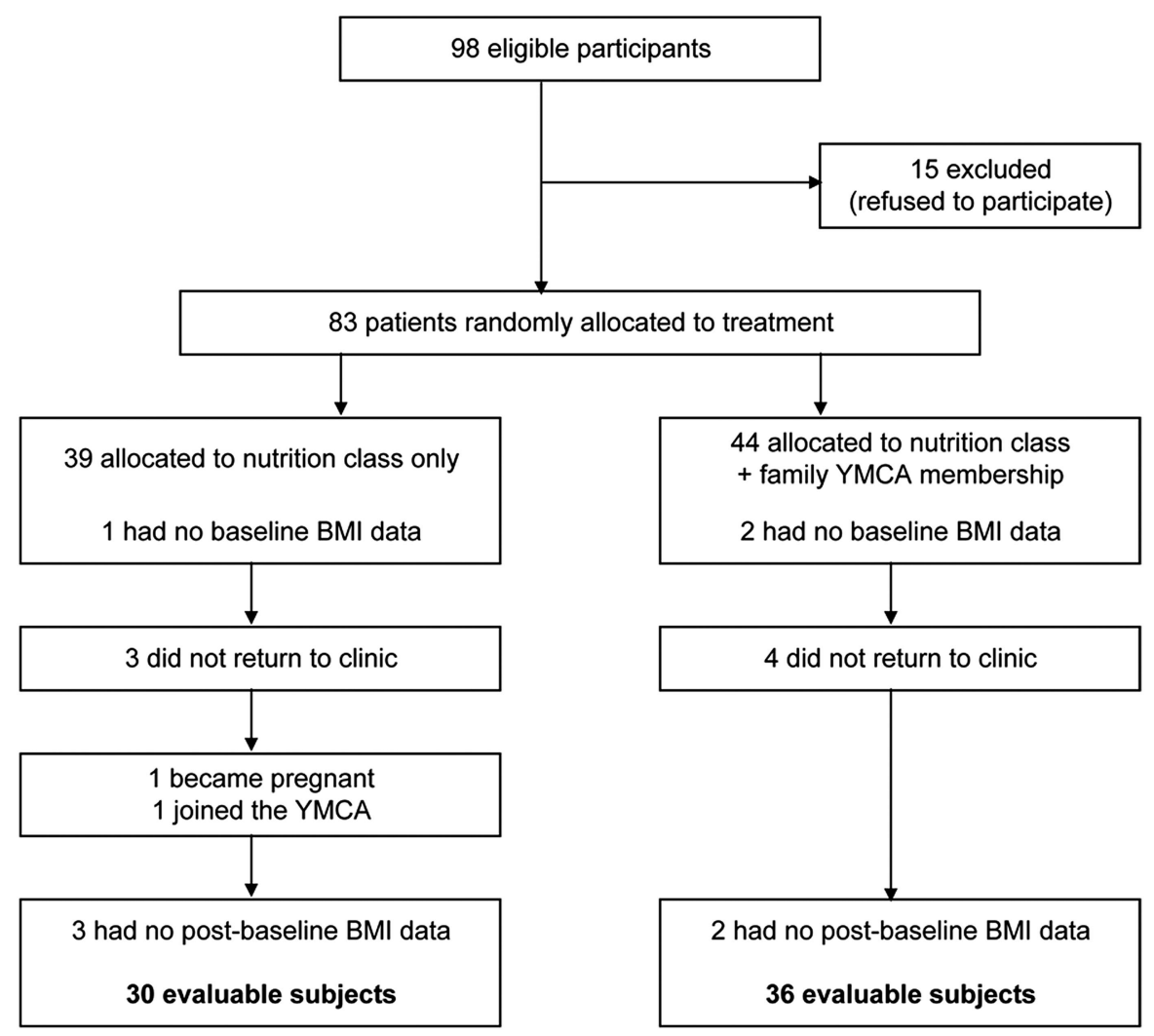

Resource limitations required that enrollment for nutrition classes be completed in 12 months and study power was chosen with dietitian availability as the limiting factor. Given a targeted change of 2 points in BMI percentile, with an $\alpha=.05, \mathrm{SD}=4$, and a power $(1-\beta)$ of .70 , a sample size of 50 per group was planned.

\section{Results}

Eighty-three children from 83 families were randomized to treatment between August 1, 2005, and January 31, 2006. As detailed in Figure 1, 17 children were excluded from analysis, leaving evaluable data from 30 children in the control group and 36 in the treatment group.

Participants were extremely obese; although inclusion criteria allowed a BMI percentile as low as 85, the median BMI percentile was 99 (see Figure 2). Most families had an annual income of less than $\$ 20,000$, and almost a quarter of parents had not completed high school. By self-report, a median of $50 \%$ of family members were overweight. No base- line differences were noted between groups on any measure (see Table 1).

Attendance at nutrition classes varied with treatment group. At least one class was attended by $67 \%$ of the treatment group, but only $30 \%$ of the controls $\left(\chi^{2}, P<.01\right)$. Of those attending, a median of 3 nutrition classes was attended by those in the treatment group compared with only 2 in the control group. Eating habit questionnaires (Table 2) were completed by 29 attendees during their initial class and by 14 during their final class. The largest shift from calorie-dense to nutrient-dense foods was reported for beverages, where food type score decreased from 2.3 (SD 0.8) to 1.5 (SD 0.7); 8 of the 12 within-subject comparisons showed improvement in beverage type. Number of daily servings decreased from 3 to 4 to 1 to 2 in the milk products and meat products groups; within-subject comparisons were consistent with the reduction in meat products but not milk products. Only 12 participants (2 in the control group and 10 in the treatment group) provided within-subject compar- 
Figure 2. Distribution of body mass index (BMI) percentile at baseline in (top) control $(n=36)$ and (bottom) treatment $(\mathrm{n}=30)$ groups.

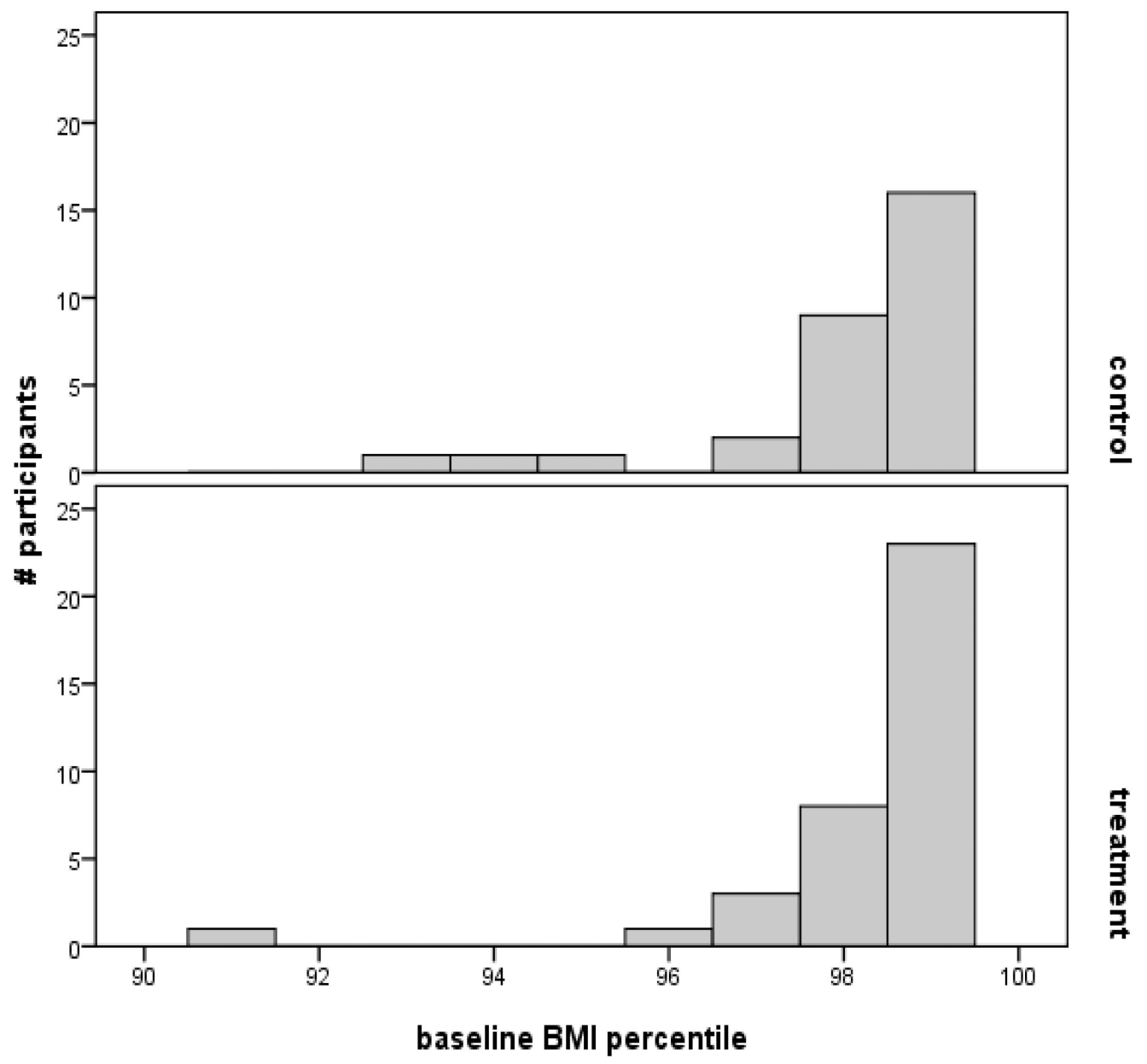

ative data, which precluded meaningful group comparisons.

Of the 36 evaluable participants randomized to the treatment group only 27 ever visited the
YMCA, with a median of 5 visits (interquartile range, 2-13) reported. For 18 reporting participants, a median of 82 minutes (interquartile range, 61-119) was spent at each visit. One or 2 family

Table 1. Characteristics of Participants

\begin{tabular}{lcc}
\hline & Control $(\mathrm{n}=30)$ & Treatment $(\mathrm{n}=36)$ \\
\hline Age, years (mean [SD]) & $10.6(3.4)$ & $10.6(3.9)$ \\
Grade, year (median) & 5 & 5 \\
Female (\%) & 60 & 42 \\
BMI percentile (median [range]) & $99.0(93-99)$ & $99.0(91-99)$ \\
Bilingual household (\%) & 20 & 11 \\
Income <\$20,000 (\%) & 80 & 69 \\
Parental high school education (\%) & 73 & 70 \\
Other household members (median) & 3 & 2 \\
Any members overweight (\%) & 90 & 82 \\
Number members overweight (median) & 1 & 1
\end{tabular}

BMI, body mass index. 


\begin{tabular}{|c|c|c|c|c|c|c|}
\hline \multirow[b]{2}{*}{ Food Type Score* (mean [SD]) } & \multirow[t]{2}{*}{$\begin{array}{l}\text { Baseline } \\
(\mathrm{n}=29)\end{array}$} & \multirow[t]{2}{*}{$\begin{array}{c}\text { Exit } \\
(\mathrm{n}=14)\end{array}$} & \multirow[t]{2}{*}{ Effect Size } & \multicolumn{3}{|c|}{ Within-Subject Comparisons } \\
\hline & & & & No Change & Improved & Worsened \\
\hline Vegetables & $1.5(0.9)$ & $1.7(1.0)$ & 0.2 & 9 & 2 & 1 \\
\hline Fruit & $1.4(0.7)$ & $1.4(0.5)$ & 0.0 & 6 & 2 & 3 \\
\hline Breads and cereals & $1.9(0.6)$ & $1.7(0.6)$ & -0.3 & 8 & 3 & 1 \\
\hline Milk products & $1.9(0.8)$ & $1.8(0.8)$ & -0.1 & 5 & 4 & 2 \\
\hline Meat products & $2.0(0.9)$ & $2.2(0.7)$ & 0.3 & 5 & 4 & 3 \\
\hline Snacks or sweets & $2.6(0.8)$ & $2.1(1.0)$ & -0.6 & 7 & 4 & 0 \\
\hline Dressings or sauces & $2.2(1.0)$ & $1.7(1.0)$ & -0.5 & 8 & 3 & 1 \\
\hline Beverages & $2.3(0.8)$ & $1.5(0.7)$ & -1.1 & 3 & 8 & 1 \\
\hline Number of servings (median) & & & & No Change & Fewer Servings & More Servings \\
\hline Vegetables & $1-2$ & $1-2$ & & 7 & 2 & 2 \\
\hline Fruit & $1-2$ & $1-2$ & & 6 & 2 & 4 \\
\hline Breads and cereals & $1-4$ & $1-4$ & & 9 & 1 & 2 \\
\hline Milk products & $3-4$ & $1-2$ & & 6 & 3 & 3 \\
\hline Meat products & $3-4$ & $1-2$ & & 7 & 5 & 0 \\
\hline Snacks or sweets & $1-2$ & $1-2$ & & 5 & 5 & 2 \\
\hline
\end{tabular}

*Within each food group, 1 = nutrient-dense, 2 = intermediate, 3 = calorie-dense.

members usually attended with the participant. The most popular activities were weightlifting (13 participants), swimming (13 participants), and walking (12 participants) followed by jogging (7 participants) and basketball (7 participants).

Four participants in the control group and one in the treatment group achieved the target reduction of 2 BMI percentile points (Fisher's exact, $P=$ .17; see Table 3). Because median baseline BMI percentile was extreme and relatively insensitive to change, multiple other measures of weight control were examined. Maintenance of BMI, the underlying concept that led to our choice of primary out- come, was achieved by 10 participants in each group (see Figure 3). Three of the participants in the control group (10\%) lost weight (3.0, 5.2, and $104 \mathrm{lbs})$. Five of the participants in the treatment group (13.9\%) lost weight (2.4, 5.0, 7.3, 8.7, and $22.4 \mathrm{lbs})$. A Mann-Whitney test indicated no statistically significant difference between groups in change in BMI or change in weight. AMA weightloss targets were met by 7 of the 30 control group participants $(23 \%)$ and 7 of 36 of the treatment group participants $\left(19 \% ; \chi^{2}\right.$ test, $\left.P=.70\right)$.

Within the treatment group, YMCA attendees had a mean increase of $0.30 \mathrm{BMI}$ points, compared

Table 3. Weight-based Measures at Baseline and End of Study

\begin{tabular}{|c|c|c|c|}
\hline & Control $(\mathrm{n}=30)$ & Treatment $(\mathrm{n}=36)$ & Effect Size \\
\hline Visits (mean, SD)* & $3.6(1.3)$ & $3.7(1.4)$ & \\
\hline \multicolumn{4}{|l|}{ BMI percentile } \\
\hline Baseline (median) & 99 & 99 & \\
\hline Change (median) & 0 & 0 & \\
\hline Lost 2 points $(n)^{\dagger}$ & 4 & 1 & -0.45 \\
\hline \multicolumn{4}{|l|}{ BMI } \\
\hline Change (mean) & +6.5 & +10.2 & -0.23 \\
\hline Maintained BMI (n) & 10 & 10 & 0 \\
\hline Lost weight (n) & 3 & 5 & +0.12 \\
\hline Met AMA weight loss targets (n) & 7 & 7 & -0.10 \\
\hline
\end{tabular}

*Number of visits (of 6 scheduled) attended by patient, with height and weight data.

${ }^{\dagger}$ No difference between groups; Fisher's exact test, $P=.17$.

BMI, body mass index; AMA, American Medical Association. 
Figure 3. Distribution of change in body mass index (BMI) in control (top) and treatment (bottom) groups.

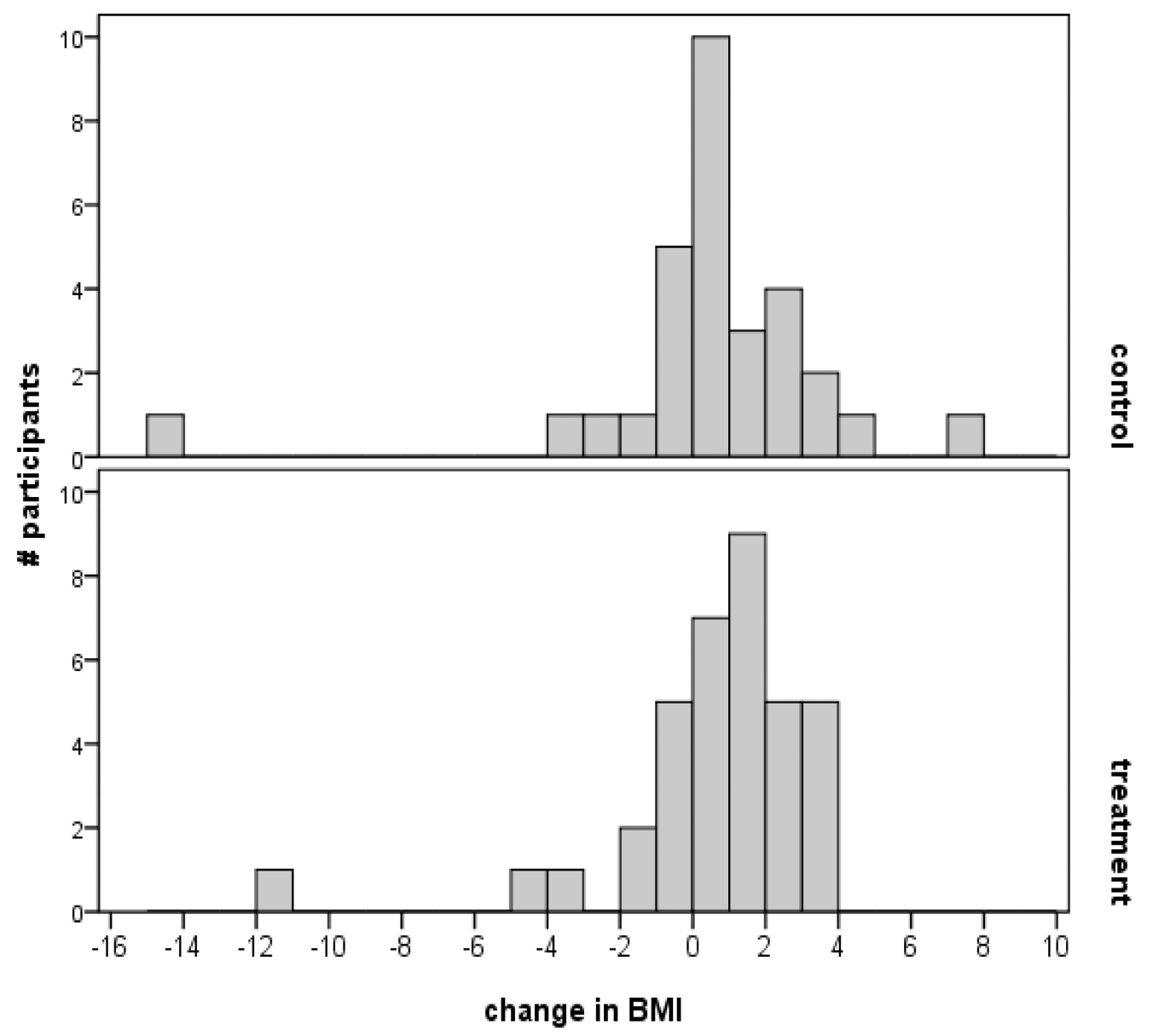

with an increase of $0.60 \mathrm{BMI}$ points among nonattendees; this change was not statistically significant $(P=.28$; see Figure 4). As shown in Figure 5 among YMCA attendees the relationship between the number of visits and the loss of either BMI or weight was positive but very small (explaining less than $10 \%$ of the variance) and not statistically significant $(P=.15$ and $P=.29$, respectively).

\section{Discussion}

This randomized, controlled effectiveness trial in a primary care setting found no evidence that provision of a 1-year, free family membership to a YMCA led to improved weight control of obese children as compared with nutrition education alone. However, those who were given the memberships attended more nutrition classes, and those who attended more YMCA sessions had more weight loss. More widespread attendance at nutrition counseling sessions may have been enhanced by reinforcement of the free membership on initial motivation to lose weight. Motivation might also underlie the positive relationship between weight control and the number of visits to the YMCA despite the lack of difference between the weight change in the control and intervention groups, which may be because of the overall low YMCA attendance rate.

This study adds to the sparse literature describing randomized, controlled treatment trials about childhood obesity conducted in primary care clinics in the United States. Urban clinics such as ours are a setting in which treatment interventions are likely to be most needed ${ }^{13}$ : in the United States, children from lower socioeconomic groups have a greater likelihood of obesity. ${ }^{14}$ The majority of research in the field has been conducted in motivated, middleclass, white populations. ${ }^{4}$

Many limitations of previous studies ${ }^{15}$ were addressed here. Few programs have involved parents, 
Figure 4. Distribution of change in body mass index (BMI) in treatment group, by attendance at YMCA.

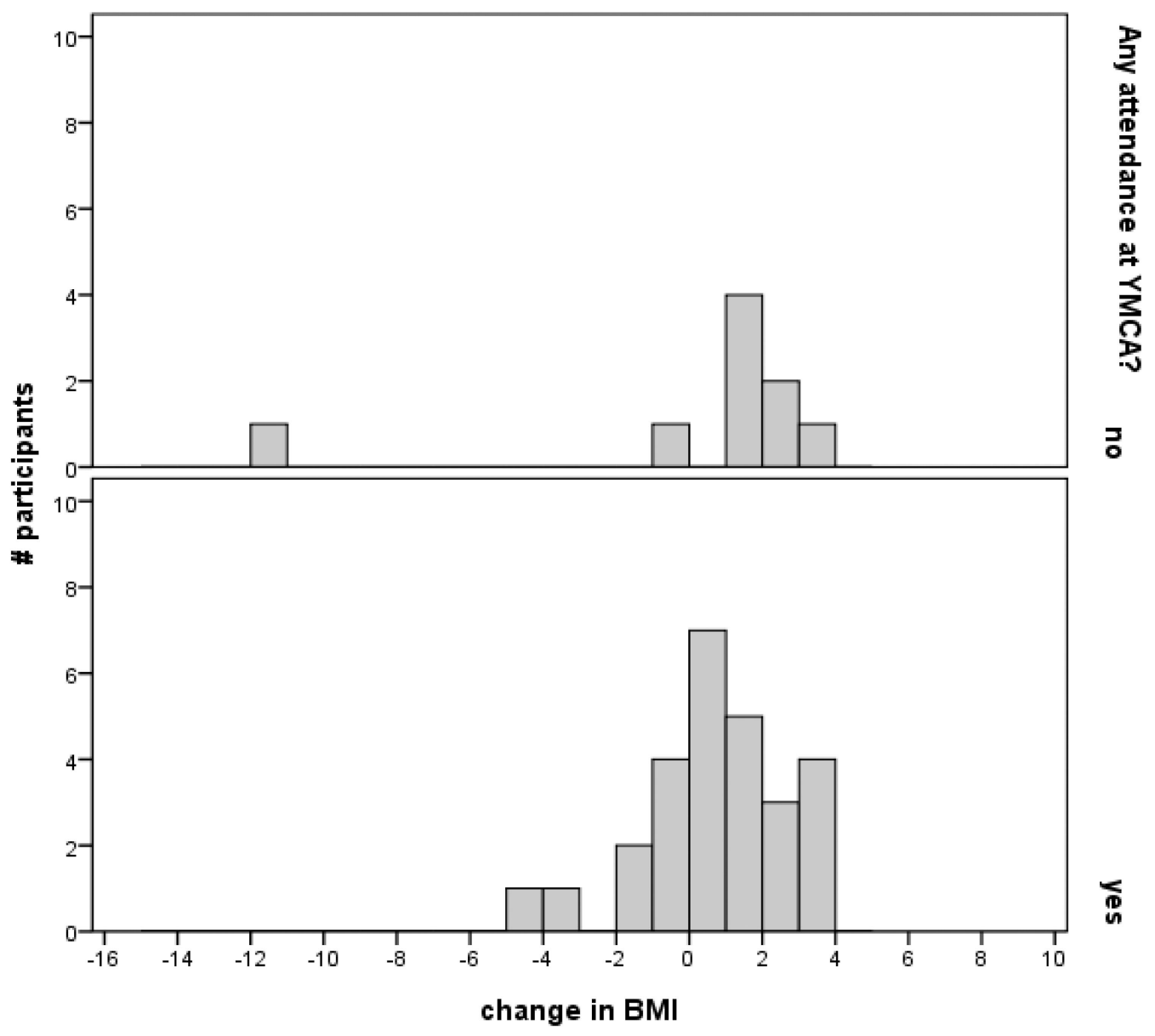

a component found to be integral to success. $\mathrm{Pa}-$ rental involvement was promoted in the current study through invitation of the entire family to attend nutrition classes and provision of a YMCA membership that could be used by the entire family. Interventions designed to change behaviors should be tested for at least 12 months because many interventions that seem to be effective in the short term (up to 3 months) are not in the long term, ${ }^{16}$ and our intervention and follow-up extended for a full year. Self-reported activity measures, which have low reliability, were confirmed through tracking by YMCA staff.

The study sample was biased toward extreme BMI percentiles. By including primarily the most extremely overweight, we might have enrolled children who have the most difficulty maintaining or losing weight. ${ }^{17}$ In such children, a targeted reduction in BMI percentile of 2 points may have been overly optimistic. Even intensive, individually tailored, daily or twice-weekly nutrition or physical activity sessions generated only a 2.8-point decrease in BMI percentile in a school-based program of children in at least the 85 th BMI percentile. ${ }^{18}$

The nutrition classes to which all participants were assigned may have improved eating habits. Dietary factors that promote obesity include highcalorie beverages, energy-dense foods, and large portion sizes. ${ }^{19,20}$ By self-report, participants shifted their beverage choices from calorie-dense (eg, regular soda) toward nutrient-dense (eg, reduced-fat milk). Ebbeling et $\mathrm{al}^{19}$ reported that decreasing sugar-sweetened beverage consumption had a beneficial effect on body weight in adolescents with $\mathrm{BMI}>25.6$. Their intervention, which was 25 weeks in duration, relied largely on home deliveries of noncaloric beverages to displace sugarsweetened beverages, and it was effective in decreasing consumption by $82 \%$ in the intervention group. BMI change was significantly more favorable in the intervention group $(-0.63 \pm 0.23 \mathrm{~kg} /$ $\left.\mathrm{m}^{2}\right)$ than in the control group $(+0.12 \pm 0.26 \mathrm{~kg} /$ 
Figure 5. Change in body mass index (BMI) (top) and weight (bottom) decreased as a function of the number of visits to the YMCA $(n=27)$.
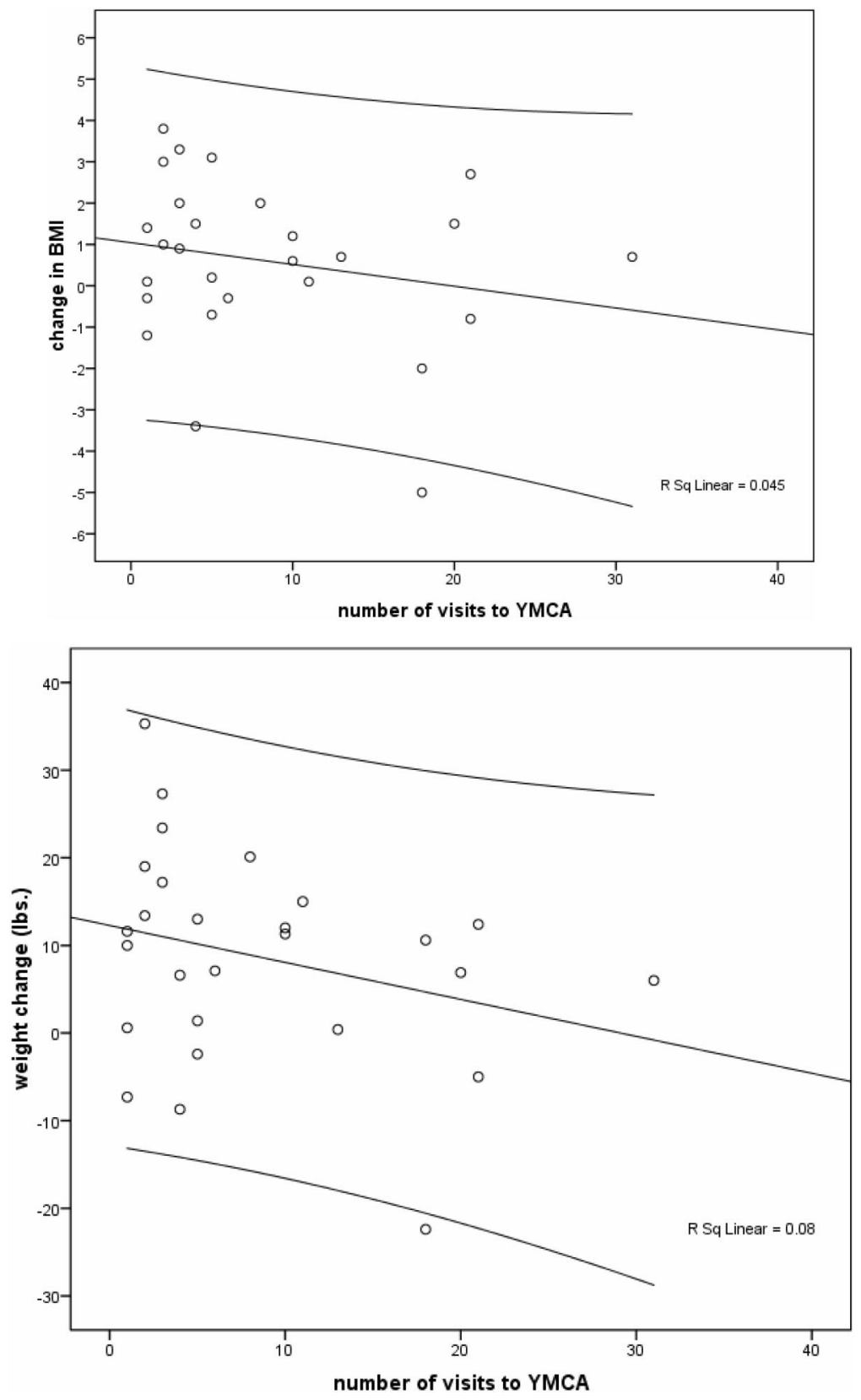

$\left.\mathrm{m}^{2}\right)$. Though based on a minority $(<25 \%)$ of participants and subject to reporting bias, our survey results suggest that the education of obese children and their families about consumption of calorie-dense beverages may be particularly efficacious in helping them achieve more healthy lifestyle habits.

Nutrition guidance alone can be effective in reducing overweight. Using nutritional charts completed by participants 3 days per month, Satoh et $\mathrm{al}^{21}$ provided 21 children with dietary guidance once per month for 6 months; 8 control children completed the chart but did not receive dietary guidance. Average weight of the intervention group decreased during the 6 months-from $50 \%$ above age- and gender-based standards to $42 \%$ abovecompared with a slight increase in the control group. Individualized rather than group education regarding nutrition may be required to achieve measurable decreases in weight. 
Recognition of obesity and subsequent motivation to change are important and related issues that were not addressed in our study. The association between obese children and obese parents is well-established, ${ }^{22}$ and eating and lifestyle patterns are largely determined by family beliefs and behaviors. Although experts stress the importance of family-based interventions, many families do not perceive the child as obese. ${ }^{23}$ Children are rarely presented for weight issues, leaving it up to the physician to identify and address them. Unfortunately, because the patients chosen for the study by residents and faculty had a median BMI percentile of 99 , patients at the 85 th percentile may have been perceived as normal weight. If the most extreme overweight are also the most difficult to treat, as noted above, frustration with efforts to treat obesity may occur. Insensitivity to obesity may also underlie the missing height and/or weight data for $10 \%$ of enrolled patients that led to their exclusion from analysis.

The key role of motivation was exemplified by the biggest loser-a young man who lost more than 100 pounds without the benefit of being randomized to the treatment group. Given that there were no group differences in weight change, motivation probably played a causal role in the relationship between the number of visits to the YMCA and weight loss, ie, the most motivated participants attended the YMCA most often and achieved some measure of weight control. Enhancement of motivation by lowering cost barriers to physical activity might also have promoted attendance at nutrition classes by those randomized to the treatment group. Future studies should take into account motivation to change.

Enrollment was unexpectedly low, and even extension of enrollment to provide 13 months of the limiting resource (nutrition classes) did not allow us to achieve the planned sample size. Another limitation was the impact on data availability because of a lack of attendance at nutrition classes, at the YMCA, and at follow-up visits with the primary care physician, which led to a loss of reliability of the data. Finally, transportation has been noted as the greatest barrier to physical activity (after expense) for patients in this socioeconomic group, ${ }^{10}$ but providing transportation to and from the YMCA or our clinics was not economically feasible.

\section{Conclusion}

The simple intervention for the treatment of childhood obesity tested here did not lead to significant changes in weight control. Future studies should address the importance of recognizing obesity where it exists, target children who do not exceed the 99th BMI percentile, attend to the role of motivation, include measures of fitness in addition to measures of weight, and lower transportation barriers to participation. Inclusion of measures from other family members should also be considered.

We gratefully acknowledge the assistance of Christine Shaffer, $\mathrm{MD}$, who facilitated involvement of residents in the residency program; Mims Wilkie, representing the YMCA; and registered dieticians Dr. D. Laura Keefer, MS, RD, LD, and Connie Niederuaer, MS, RD, LD.

\section{References}

1. National Center for Health Statistics. Health, United States, 2008 (DHHS 2009-1232). Hyattsville, MD: National Center for Health Statistics; 2009.

2. Daniels SR, Arnett DK, Eckel RH, et al. Overweight in children and adolescents: pathophysiology, consequences, prevention, and treatment. Circulation 2005;11:1999-2012.

3. Must A. Does overweight in childhood have an impact on adult health? Nutr Rev 2003;61:139-42.

4. Oude Luttikhuis H, Baur L, Jansen H, et al. Interventions for treating obesity in children. Cochrane Database Syst Rev 2009;(1):CD001872.

5. Daniels SR, Jacobson MS, McCrindle BW, Eckel RH, Sanner BM. American Heart Association Childhood Obesity Research Summit: executive summary. Circulation 2009;119:2114-23.

6. Epstein LH. Methodological issues and ten-year outcomes for obese children. Ann N Y Acad Sci 1993;699:237-49.

7. Schwingshandl J, Sudi K, Eibl B, Wallner S, Borkenstein $M$. Effect of an individualized training program during weight reduction on body composition: a randomized trial. Arch Dis Child 1999;81:426-8.

8. Barlow SE, Expert Committee. Expert committee recommendations regarding the prevention, assessment, and treatment of child and adolescent overweight and obesity: summary report. Pediatrics 2007;120(Suppl 4):S164-92.

9. US Preventive Services Task Force. Screening and interventions for overweight in children and adolescents: recommendation statement. Pediatrics 2005; 116:205-9.

10. Centers for Disease Control and Prevention. Physical activity levels among children aged $9-13$ years, 
United States, 2002. MMWR Morb Mortal Wkly Rep 2003;52:785-8.

11. Centers for Disease Control and Prevention. Healthy weight-it's not a diet, it's a lifestyle! About BMI for children and teens. Available at http://www. cdc.gov/nccdphp/dnpa/healthyweight/assessing/bmi/ childrens_BMI/about_childrens_BMI.htm. Accessed 16 October 2008.

12. National Institutes of Health. We can! Go, slow, and whoa foods. Available at http://www.nhlbi.nih. gov/health/public/heart/obesity/wecan/downloads/ go-slow-whoa.pdf. Accessed 15 October 2009.

13. Caprio S. Treating child obesity and associated medical conditions. Future Child 2006;16:209-24.

14. Gordon-Larsen P, Adair LS, Popkin BM. The relationship of ethnicity, socioeconomic factors, and overweight in US adolescents. Obes Res 2003;11: 121-9.

15. Small L, Anderson D, Melnyk BM. Prevention and early treatment of overweight and obesity in young children: a critical review and appraisal of the evidence. Pediatr Nurs 2007;33:149-52, 155-61, 127.

16. Glenny A, O'Meara S, Melville A, Sheldon TA, Wilson C. The treatment and prevention of obesity: a systematic review of the literature. Int J Obes 1997;21:715-37.

17. Dorsey KB, Wells C, Krumholz HM, Concato J.
Diagnosis, evaluation, and treatment of childhood obesity in pediatric practice. Arch Pediatr Adolesc Med 2005;159:632-8.

18. Johnston CA, Tyler C, McFarlin BK, et al. Weight loss in overweight Mexican American children: a randomized, controlled trial. Pediatrics 2007;120: e1450-7.

19. Ebbeling CB, Feldman HA, Osganian SK, Chomitz VR, Ellenbogen SJ, Ludwig DS. Effects of decreasing sugar-sweetened beverage consumption on body weight in adolescents: a randomized, controlled pilot study. Pediatrics 2006;117:673-80.

20. Ebbeling CB, Garcia-Lago E, Leidig MM, SegerShippee LG, Feldman HA, Ludwig DS. Altering portion sizes and eating rate to attenuate gorging during a fast food meal: effects on energy intake. Pediatrics 2007;119:869-75.

21. Satoh A, Menzawa K, Lee S, Hatakeyama A, Sasaki H. Dietary guidance for obese children and their families using a model nutritional balance chart. Jpn J Nurs Sci 2007;4:95-102.

22. Moran R. Evaluation and treatment of childhood obesity. Am Fam Physician 1999;59:861-8.

23. Young RF, Schwartz KL, Monsur JC, West P, Neale AV. Primary care of overweight children: the importance of parent weight and attitudes about overweight: a MetroNet study. J Am Board Fam Med 2008;21:361-3. 